\title{
Spectro-Photo-Interferometry of Stellar Pulsation (SPIPS)
}

\section{A more precise and more accurate implementation of the parallax of pulsation method}

\author{
Antoine Mérand $^{1, \star}$, Pierre Kervella ${ }^{2,3,4}$, Alexandre Gallenne ${ }^{1}$, Joanne Breitfelder ${ }^{1}$, \\ Boris Trahin ${ }^{2,3,4}$, and Marion Villenave ${ }^{5}$ \\ ${ }^{1}$ European Southern Observatory, Alonso de Córdova 3107, Casilla 19001, Santiago 19, Chile \\ ${ }^{2}$ LESIA (UMR 8109), Observatoire de Paris, PSL, CNRS, UPMC, Univ. Paris-Diderot, 5 place Jules Janssen, \\ 92195 Meudon, France \\ ${ }^{3}$ Unidad Mixta Internacional Franco-Chilena de Astronomía, CNRS/INSU, France (UMI 3386), Departa- \\ mento de Astronomía, Universidad de Chile, Camino El Observatorio 1515, Las Condes, Santiago, Chile \\ ${ }^{4}$ Universidad de Concepción, Departamento de Astronomía, Casilla 160-C, Concepción, Chile \\ ${ }^{5}$ Institut Supérieur de l'Aéronautique et de l'Espace, Toulouse, France
}

\begin{abstract}
We present our implementation of the parallax of pulsation method which integrates all observables and physical modelling of the photosphere to get the best statistical precision and controlled biases. This method has been validated on well known stars and used to estimate observationally the projection factor of the HST-FGS sample. Our future developments include application to the Gaia Cepheids and modelling of the spectrum.
\end{abstract}

\section{Why revisit the parallax of pulsation method?}

The parallax of pulsation (PoP) method compares the integrated pulsation velocity and angular diameter variations of pulsating stars, in order to derive the distance. The PoP method is the "most geometrical" way, apart from measuring the direct parallax, to estimate distance to Cepheids. As such, the method is central in calibrating the Leavitt's (period-luminosity) law.

Traditional implementation (so called Baade-Wesselink) have lots of limitations which affect the accuracy and precision of the derived distances.

First, pulsation velocity measured using spectroscopy does not represent exactly the pulsation velocity: the use of an ad-hoc projection factor to estimate the pulsation velocity from the radial velocity introduces a bias to the distance. This p-factor depends on the extraction method of radial velocity, which makes the bias complicated to account for in practice.

Secondly, angular diameters are usually derived using 2 bands photometry (such as $\mathrm{V}$ and $\mathrm{K}$ ): two parameters ( $T_{\text {eff }}$, diam) are actually adjusted from two measurements, which is an under-use of multiple bands usually available (up to a dozen different). Surface brightness laws need to be calibrated, and use a simplistic linear dependency with the color. Moreover, reddening and reddening law need to be known. Note that so-called Wesenheit relations (a linear combination of 2 magnitudes) is not immune to that since the linear relation is derived from reddening laws.

^amerand@eso.org 


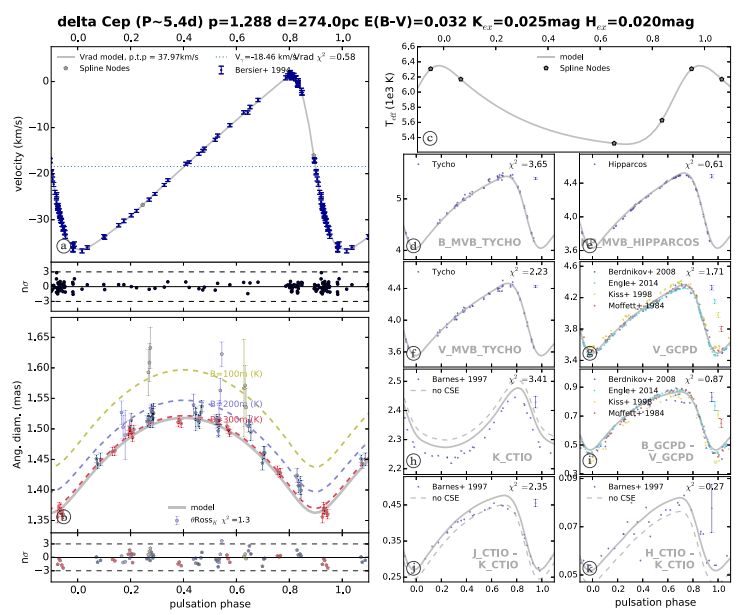

Figure 1. SPIPS fit for $\delta$ Cep: velocity, angular diameter and photometric fit. Note the infrared excess (in $\mathrm{K}$ band photometry) as well as the bias in the apparent angular diameter.

Finally, the traditional Baade-Wesselink implementation uses non optimal data combination schemes. Usually, data are fitted in various stages, i.e. not at once, which render the final uncertainties too optimistic. Too often, low order interpolation is used which leads to poor statistical significance and biases to the distance. Additionally, only all-at-once data combination allows to efficiently combine data with poor phase coverage and/or account for period change.

\section{A better approach}

The PoP method is not geometric. We embrace its complexity and dependence on the pulsation's physics. Our approach to improve precision and accuracy is as follows. Combination of all data possible: spectroscopic radial velocities; high resolution spectra; any number of photometric measurements (not limited to 2 bands); optical interferometric measurements. Simultaneous fit of ALL the parameters: 2 profiles as function of time for $\operatorname{Vpuls}(t)$ and $\operatorname{Tef} f(t)$; period and period change parameters; reddening $E(B-V)$; distance or p-factor. Use of atmospheric models to derive synthetic photometry from the effective temperature. Inclusion of morphological and photometric modelling of infrared excesses.

This approach allowed us to successfully model all observables from 2 stars in great details ( $\delta$ Cep and $\eta$ Aql, the first of which can be seen on Fig. 1) which proves the validity of the approach [1]. Our future work includes high resolution spectroscopic modelling, as well as extension of SED modelling beyond the K-band. Other uses of SPIPS include the modelling and derivation of p-factor for the Type II Cepheid $\kappa$ Pav ([2]), the HST-FGS Cepheids sample ([3]) and for the long period Cepheid RS Pup ([4]). All these examples of SPIPS modelling of Cepheids with available distances point toward a constant p-factor of the order of 1.25 to 1.30 , regardless of the pulsation period (see Kervella et al., these proceedings).

\section{References}

[1] Mérand, A., Kervella, P., Breitfelder, J., et al., A\&A, 584, 10 (2015)

[2] Breitfelder, J., Kervella, P., Mérand, A., et al., A\&A, 576, 64 (2015)

[3] Breitfelder, J., Mérand, A., Kervella, P., et al., A\&A, 587, 117 (2016)

[4] Kervella, P., Trahin, B., Bond, H. E., et al., A\&A , 600, A127 (2017) 